\title{
Inhalt vom Band 260
}

\section{Heft 1}

Austegeben im Februar 1979

B. Tökes, É. GyArfís and L. KékedY: Polarographic Study of Acidbase Equilibria of Benzoic Acid and Some Derivatives in Aqueous-alcoholic Solutions. With 1 Fig. and 2 Tab. (Received 18. 4. 77)

A. Lycourghiotis and A. Tsiatsios: Adsorption Properties of Alumina Modified with Lithium Chloride. With 5 Fig. and 4 Tab. (Received 21. 3. 77)

A. A. Fakhry, A. NaSr Alla and M. S. Hashem: Effect of Spectroscopic Buffers on Transite Time $\tau$ and Diffusion $D$ of Atoms in DC Arc Discharge. With 2 Fig. and 1 Tab. (Received 28. 1. 77) .

S. S. AbD El ReHm and W. H. Boctor: Effect of Some Anions on the Anodic Behaviour of $\mathrm{Pb}$ in $\mathrm{H}_{2} \mathrm{SO}_{4}$ Solutions. With 5 Fig. (Received 9. 3.77)

W. C. Vasudeva and M. R. Suliman: A Kinetic Study of the Reaction of Fumaric Acid with Peroxydisulphates. With 1 Fig. and 3 Tab. (Received 29.4. 77)

A. Kumar, A. K. Bose, R. M. Mehrotra and S. P. Mushran: Kinetics and Mechanism of the Osmium Tetroxide Catalysed Oxidation of Cyclopentanone by Chloramine-T. With 1 Fig. and 3 Tab. (Received 15.11. 76, in rev. version 19. 5.77)

T. S. RaO, S. I. MaLI and V. T. Dangat: Kinetics of the Rapid Reaction $\mathrm{Br}_{2}+2 \mathrm{I}^{-} \rightarrow \mathrm{I}_{2}+2 \mathrm{Br}^{-}$ in Aqueous Solution. With 2 Tab. (Received 21.3. 77, in rev, version 21. 7. 77) . . . . .

R. SALZER: Untersuchung der Bandenform der OH-Valenzschwingung homologer n-Alkohole. Mit 3 Abb. und 1 Tab. (Eing. 29. 10. 76, in rev. Fassg. 5. 8. 77)

M. Scholz und E. WALzel: Chemie angeregter Zustände. VII. Mitt.: Quantenchemische Untersuchungen am $\pi$ - $\pi$-Molekülkomplex Azulen-Tetracyanoäthylen (TCNÄ). Mit 2 Abb. und 6 Tab. (Eing. 27.6 77; in rev. Fassg. 16.8.77)

H.-J. BrtTrich, B. KillaN und K. Geter: Untersuchungen zur selektiven Stofftrennung. IV. Kritische Mischungstemperaturen von Kohlenwasserstoffen mit Dimethylformamid. Mit 3 Abb. und

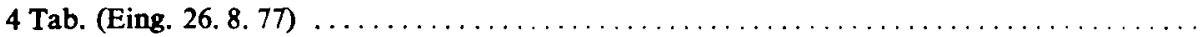

A. K. Helmy and N. Peinemann: Sorption of Fluoride by Hydrous Aluminium Oxide. With 2 Fig. (Received 21. 7. 77)

R. L. Mishra and J. D. Pandey: Non-linearity and Related Parameters for Liquid Xenon. With 1 Tab. (Received 8. 8. 77)

C. E. Chellam and G. Aruldhas: An Iterative Parameter Method for Calculation of Unique Force Constants. With 1 Tab. (Received 12. 8. 77)

J. FABIAN: MO-LCAO-Berechnungen an schwefelhaltigen --Elektronensystemen. 41. Mitt.: Bewertung alternativer $\pi$-Modelle für konjugierte Heterocyclen. Mit 3 Abb. und 2 Tab. (Eing. 28. 6. 77 ;

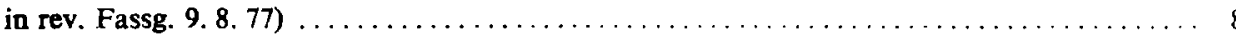

M. Fischer: Uber die Funktion des Oxydationsmittels bei der chemischen Passivierung des Eisens.

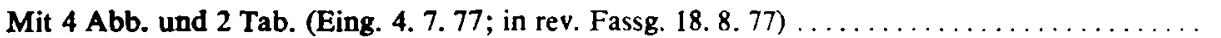

H. ANDERSON, W. Besch und D. HABerLand: Vergleichende Untersuchung zu Verfahren der kinetischen Auswertung von DTA-Kurven. Mit 8 Abb. und 2 Tab. (Eing. 25. 8. 77) 
J. Bendig, D. Kreysig und L. Meinicke: Desaktivierungsverhalten von Arenen und Heteroarenen. VII. Änderung der spektroskopischen Eigenschaften durch Assoziatbildung bei hohen Konzentrationen. Mit $6 \mathrm{Abb}$. und 1 Tab. (Eing. 19.9. 77)

M. Fischer: Uber korrosionshemmende Zusätze für Eisen im Neutralbereich. Ergebnisse systematischer Untersuchungen der passivierenden Wirkung luftgesāttigter wāßriger Lösungen von Salzen aliphatischer Carbonsäuren. Mit $1 \mathrm{Abb}$. und 2 Tab. (Eing. 21. 9. 77)

D. Hartmann, G. Herzog und K. Schellmann: Luminophore auf der Basis aktivierter ZinkYttrium-Mischoxide für die Anwendung in Cadmiumdampf-Niederdruckentladungslampen. III. Zum Einfluß einwertiger Kationen auf Emission und Anregung Bi-Eu- und Bi-Dy-biaktivierter Leuchtstoffe des Grundgitters $0,4 \mathrm{~mol} \mathrm{ZnO}+0,6 \mathrm{~mol} \mathrm{Y}_{2} \mathrm{O}_{3}$. Mit $8 \mathrm{Abb}$. und 3. Tab. (Eing.

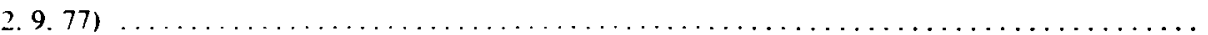

K. SüHNEL, L. MESSOW und M. SALOMON: Thermodynamische Untersuchungen an Lösungsmittel' n-Paraffin-Systemen. X. Die Anwendung des Gittermodells von Barker zur Vorausberechnung der thermodynamischen Exzeßeigenschaften in Keton, n-Alkan-Systemen. Mit 3 Abb. und 4 Tab. (Eing. 22. 3. 77; in rev. Fassg. 14. 8. 77)

J. BeNDig und D. KreYsig: Desaktivierungsverhalten von Arenen und Heteroarenen. VIII. Reabsorption und Reemission bei frontaler Fluoreszenzanregung. Mit $13 \mathrm{Abb}$. und $5 \mathrm{Tab}$. (Eing. 19. 9. 77)

\section{Kurze Mitteilungen}

P. N. Tiwari, S. C. Ameta and V. F. Shastry: Kinetics of (oxidation of Acetic Acid by Potassium

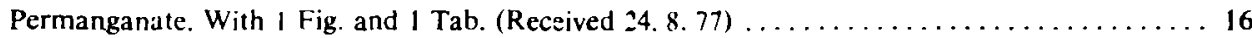

G. Kolasinśa and P. Oracz: Generalization of Error Analysis and Problem of Vapour NonIdeality in Barker's Method of Vapour Pressure Isotherm I)ata Processing. With 4 Tab. (Received 24. 1. 78)

H. BrandL: Zur Geschichte des Einheitskompressibilitätsgesetzes (Unit Compressibility Law). (Eing. 27. 3. 78)

S. Sokozowsk1: A Note on the Local Density of Hard Spheres Interacting with a Hard Wall. With

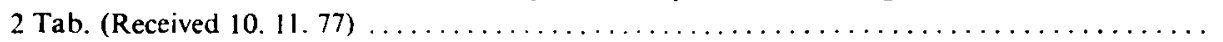

O. Herbarth und F. Plümer: Untersuchungen zur strahlungsinitierten Polymerisation. III. Beweglichkeit ionischer Intermediate bei der Polymerisation von Styrol im Bereich geringer Umsätze. (Eing. 5. 4. 78)

B. A. Hess Jr. and L. J. SCHAAD: On the Relation Between $\pi$-Binding Energy and Bond Orders. With 2 Tab. (Received 25. 5. 78)

H. Kehlen, R. Pulss und L. Vogel: Das Verhalten binärer flüssiger Mischphasen. XI. Die Mischungsenthalpien in Systemen aus Cyclohexan und Zinntetraalkylen. Mit 4 Tab. (Eing. 14. 6. 78) 186

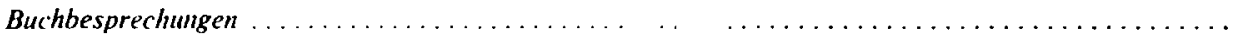

Berichtigung

\section{Heft 2}

Ausgegeben im Mas 1979

P. K. Mandal, B. K. Seal and A. S. Basu: Studies on the Viscosity of Mixtures of Electrolytes in Aqueous Solution. II. The Viscosity Contribution Coefficients of the Electrolytes in Presence of Each Other. With 4 Fig. and I Tab. (Received 4. 2. 77) 
S. K. JAIN and H. C. GAUR: Electrical Conductivity of Molten Mixtures of Potassium Nitrate with Lead Nitrate and the Tobolsky Parameter. With 3 Fig. and 3 Tab. (Received 27. 11. 76; in rev. version 30. 6. 77)

D. K. Sharme, U. P. Verma and A. N. Pandey: A New Approach Towards Exact Force Field Calculations For $\mathbf{N}=3$ Eigen-value Problem Using L-F Approximation and HLFS Method and Application to Some XYZ Bent Type Molecules. With 2 Tab. (Received 14. 9. 77)

R. P. Varma and H. K. Bhargava: Conductance Behaviour of Iron and Cobalt Soaps of Lower Fatty Acids in 2-methyl-1-propanol. With 4 Fig. and 5 Tab. (Received 3. 10. 77)

M. JARoniec, A. Patrykmejew and M. Borówko: Some Remarks on the Langmuir Adsorption Isotherm. With 1 Fig. (Received 16.9. 77)

B. Thomas, G. Seirert, G. Grossmann und D. Scheller: NMR-spektroskopische Untersuchungen an ${ }^{15} \mathrm{~N}$-markierten Chlorocyclophosphazenen. Mit 4 Abb. und 6 Tab. (Eing. 8. 7. 77; in rev.

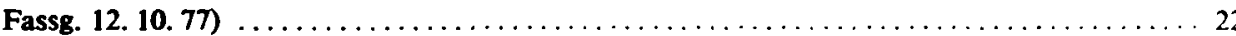

W. Ploss, D. Freude und H. Pferfer: Untersuchungen zur Deuterierung von Zeolith-Hydroxyl-

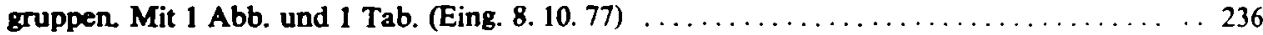

W. LoRENz: Molekulare Modelle in kondensierten Systemen und an Grenzflächen. (Eing. 15. 10. 77) 241

R. Hartung: Polarisationsverhalten und Wechselstrombehandlung einer $\mathbf{P t} / \mathbf{Z r}_{\mathbf{0 . 8 2}} \mathbf{Y}_{\mathbf{0 . 0 1}} \mathbf{M g}_{0.08}$ $\mathrm{O}_{1,87} \mathrm{O}_{2}$-Elektrode. Mit $8 \mathrm{Abb}$. und $1 \mathrm{Tab}$. (Eing. 22. 7. 77; in rev. Fassg. 23. 10. 77) „... 259

R. D. Singh: Interaction Potential for the Atoms and the 100 Face of Simple (ubic Alkali Halides.

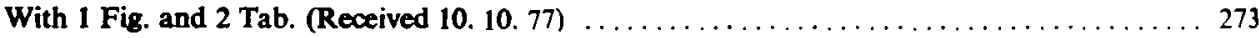

H.-H. Emons, P. Hauptmann und D. Pohl: Ultraschallgeschwindigkeits- und Ultraschallabsorptionsmessungen an ausgewählten Elektrolytlösungen. Mit $4 \mathrm{Abb}$. und 1 Tab. (Eing. 11.11. 77) 281

H. Dautzenberg und B. Phin.rp: Kinetische Untersuchungen zur Hydrolyse des Mono- und Dichloracetats in alkalischem Medium. Teil I: Problemstellung und experimentelle Ergebnisse für Monochloracetat. Mit 3 Abb. und 4 Tab. (Eing. 20. 10.77)

H. Dautzenberg und B. Phultpp: Kinetische Untersuchungen zur Hydrolyse des Mono- und Dichloracetats in alkalischem Medium. Teil 1I: Untersuchungen an Di- und Trichloracetat und zusammenfassende Diskussion der Ergebnisse. Mit 3 Abb. und 5 Tab. (Eing. 20. 10. 77)

B. S. MahL, J. R. Khurma, M. S. Dhillon and Z. S. Kooner: Excess Enthalpies and Weak Interactions of o-Dichlorobenzene with Aromatic Hydrocarbons. With 2 Tab. (Received 31. 10. 77) 309

J. Bendig, C. Csongar und D. KreYsig: Desaktivierungsverhalten von Arenen und Heteroarenen. IX. Reabsorption und Reemission bei in-line-Fluoreszenzanregung mit Dreieckküvette. Mit 5 Abb. und 1 Tab. (Eing. 8. 11. 77)

K. Maser, J. Dubnack und R. Jux: Siliziumreinigungseffekt während der $\mathrm{POCl}_{3}$-Trägergasdiffusion. Mit $5 \mathrm{Abb}$. und $4 \mathrm{Tab}$. (Eing. 10. 10.77)

M. TschapeK, C. Wasowski and R. M. Torrfs Sanchez: Competitive Adsorption of Ions and Neutral Organic Molecules on Hydrophobic and Hydrophilic Surfaces. With 10 Fig. and 1 Tab.

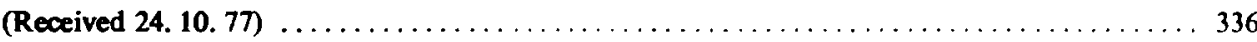

A. KÖFLER: Strukturumwandlungen nematischer Flüssigkristalle am Phasenübergang nematischisotrop. Mit 13 Abb. (Eing. 8. 11. 77)

A. Wlegeleben, J. Deresch, D. Demus und I. I Konstantinoff : Limwandlungsenthalpien kristallin-flüssiger 4n-Alkyloxy-benzoesāure-[4-n-acyl-phenylester]. Mit $1 \mathrm{Abb}$. und 1 Tab. (Eing.

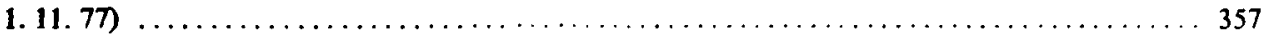

U. Jensen und H.-W. Uhlig: Der Entladungsmechanismus der $\mathrm{MnO}_{2}$-Elektrode. Mit $8 \mathrm{Abb}$. und 3 Tab. (Eing. 23. 11.77) 
I. Kruk, K. Lichszield and T. Michalska: The Extra-Weak Spontaneous Chemiluminescence Decomposition of Hydrogen Peroxide. With 2 Fig. (Received 19. 10. 77)

B. Nagel, R. Binner und J. Fruwert: Fermiresonanz-Parameter von Methyljodid, Methyljodid-d 3

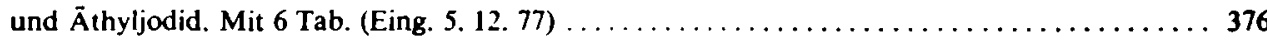

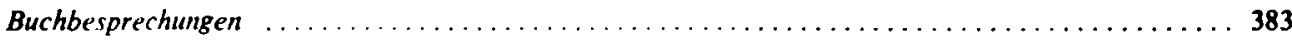

\section{Heft 3}

Ausgegeben im Juni 1979

Glückwunsch für Prof. Dr. phil. Dr. rer. nat. h. c. Dr. rer. nat. h. c. P. A. Thiessen zur Vollendung seines 80. Lebensjahres. Mit 1 Abb.

H. Thamm, Ch. Morgeneyer und W. Schirmer: Zur Berechnung von Adsorptionspotentialen

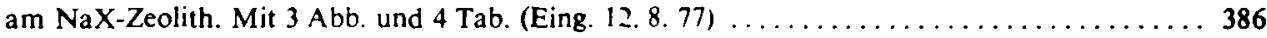

H. Blank, M. Bílow and W. Schirmer: Special Features of the Adsorption Equilibria of the

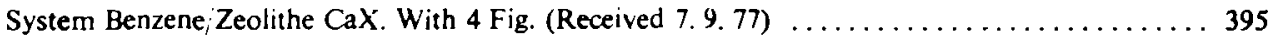

K. P. Thiessen: Energetische Randbedingungen tribochenischer Prozesse. I. Stufenschema der Energie-,,Zustände" tribochemischer Prozesse. Mit 1 Abb. (Eing. 9. 1. 78) . ...........403

K. P. Thiessen und K. SIEBtR: Energetische Randbedingungen tribochemischer Prozesse. II. „Tribochemische Effekte" Stationäre Zustände n-ter Ordnung. (Eing. 9. 1. 78) . ..........410

K. P. Thiessen und K. Sieber: Energetische Randbedingungen tribochemischer Prozesse. III. Hypothetische Geschwindigkeits'Affinitätsbeziehung tribochemischer Prozesse. Mit 4 Abb. (Eing. 9. 1. 78)

L. KUHNERT und H. LINDE: Signal-Antwort-Verhalten von autokatalytischen Reaktionssystemen. I. Auslösung von Oszillationen, Proportional-Differential-Antwortverhalten und Refraktarität bei photochemischen Reaktionssystemen. Mit 10 Abb. (Eing. 18. 4. 78)

H.-J. KRUG, L. KUHNERT und H. LiNDE: Signal-Antwort-Verhalten von autokatalytischen Reaktionssystemen. II. Analyse der Reaktion eines autokatalytischen Systems auf einen Rechteckreiz. Mit 6 Abb. (Eing. 18. 4. 78)

E. Letrel, A. Pinkowskı und K. P. Thiessen: Einfluß einer mechanischen Prallkörperbearbeitung auf die Diffusionsverhältnisse an einer rotierenden Scheibenelektrode. Mit 3 Abb. und 2 Tab. (Eing. 27. 3. 78)

H. Stach, S. P. Shdanov, K. Fiedler, W. Schirmer und N. N. Samulevic: Das Adsorptionsgleichgewicht von n-Butan an den Zeolithen $\mathrm{NaX}$ und $\mathrm{NaY}$. Mit $8 \mathrm{Abb}$. und $2 \mathrm{Tab}$. (Eing. 21. 7. 78)

G. Winkelma.N und H.-J. SPavgenberg: Zur Verteilung von zweiatomigen Radikalen bei der Wandlung von Methan im Plasmastrahl. Mit 8 Abb. und 4 Tab. (Eing. 5. 5. 78) ........ 46s

A. K. Helmy, I. M. Natale and M. E. Ma.jdolest: Freezing Point Depression of Water in Clay

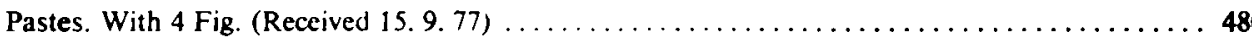

V. Y. Kabanov, K.-H. KRauss und W. ReicherdT : Elektronenreaktionen in organischen Festkörpern - RTL- und Absorptionsuntersuchungen am System PVC/Lösungsmittel nach $\boldsymbol{\gamma}$-Bestrahlung bei $77 \mathrm{~K}$. Mit $5 \mathrm{Abb}$. (Eing. 12. 7. 77; in rev. Fassg. 24.11.77) .............. 489

M. S. Dhillon and H. S. Chugh: Surface Tensions and Surface Heats of Mixing of Mixtures of o-Dichlorbenzene with Cyclohexane, Benzene. o-Xylene, m-Xylene, and p-Xylene at 293.15 and

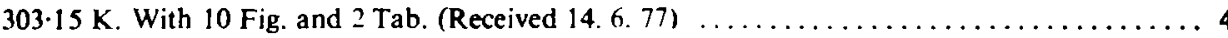


M. S. Dhillon, H. S. Chugh and S. Sivinih : Excess Volumes of Mixtures of Acetone, Chloroform, Carbon tetrachloride, 1,4-Dioxane, Tetrahydrofuran with 1,2-Dibromoethane. With 5 Fig. and 4 Tab. (Received 14, 6. 77)

V. RUth, S. All und V. B. TARE: Kinetics and Mechanism of the Reduction of Manganese Oxides in Hydrogen/Water Vapor Atmospheres. With $10 \mathrm{Fig}$. (Received 20.5.77; in rev. version 7. 12. 777

P. O. I. Virtanen, R. Korhonen and M. Alasaari: Tropylium Ions. Part IX. Environmental Effects upon the Reactions of Tropylium Ions. With $5 \mathrm{Tab}$. (Received 30.11.77)

H. Matschiner, H. TANNeberg und H.-H. Rüttinger: Zur Elektroreduktion von 1,3-Diketonatkomplexen des Kobalts. Mit 3 Abb. und I Tab. (Eing. 25. 6. 77; in rev. Fas 2 ' 10. 12.77)

A. Barth, G. Geiseler, J. Hoffmann und K. Scherzer: Zum Mechanismus der Butenisomerisierung an $\mathrm{Al}_{2} \mathrm{O}_{3}-\mathrm{Cr}_{2} \mathrm{O}_{3}-\mathrm{K}_{2} \mathrm{O}-$ Katalysatoren. $\mathrm{Mit} 6 \mathrm{Abb}$. und $5 \mathrm{Tab}$. (Eing. 22. 9. 77; in rev.

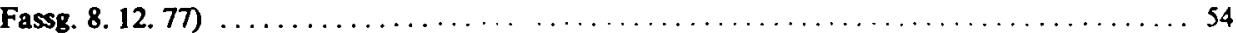

D. Klemm, E. Klemm, A. Graness und J. Kl.einschimit: Nanosekunden-Laserabsorptionsspektroskopie an $2-\left(2^{\prime}, 4^{\prime}\right.$-Dinitrobenzyl)-pyridin und 2,2',4,4'-Tetranitrodiphenylmethanen. Mit $3 \mathrm{Abb}$.

A. PACKTER: Kinetics of the Heterogeneous Reaction of Quarty Powders with Aqueous Sodium Hydroxide Solutions at $70^{\circ}$ to 115 C. With 4 Fig. and 1 Tab. (Received 11. 7. 77; in rev. version

R. Trabrtzsch und J. EpPerlein: Zur Restımniung von Quantenausbeuten für eirfache Fotoreaktionen in festen Schichten. (Eing. 20. 12. 77)

H.-D. Ilge: Beschreibung eines Meliverfahrens zur Auswertung des Extinktions- und Konzentrations-Zeitverlaufes bei Photoreaktionen mit Hilfe vollständig integrierter Geschwindigkeitsgleichungen. Mit 5 Abb. und 1 Tab. (Eing. 4. 10. 77; in res. Fassg. 29. 12. 77)

Kurze Mitteilungen

A. A. Pribylov, D. Freude und H. Schmifdel : NMR-Untersuchungen der Adsorption von $\mathrm{CFCl}_{3}$

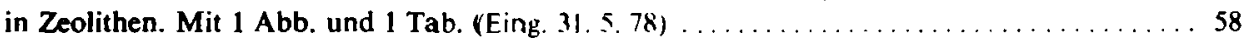

G. Dompi, D. Michel und W. MeILeR: ${ }^{13} \mathrm{C}$-NMR-Untersuchungen an Isobutenmolekülen, die in CaNaY-Zeolithen adsorbiert sind. Mut 2 Abb. und I Tab. (Eing. 9. 8. 78)

R. Kümmel und G. WILDE: Isopiestische Bestimmung der osmotischen Koeffizienten uäßriger Magnesiumnitratlösungen bei $25^{\circ} \mathrm{C}$. Mit 2 Tab. (Eıng. 3. $\left.{ }^{-} .78\right) \ldots \ldots \ldots \ldots \ldots \ldots \ldots$

K. MASER und U. SIEgEL: Röntgenmikroanalyen von Phosphor-Silizium-Phasen auf phosphor-

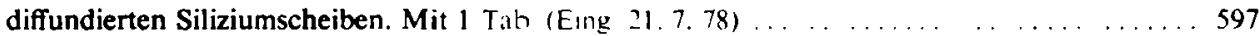

Buchbesprechungen

\section{Heft 4}

Ausgegeben im August 1979

T. Oskova und L. L. Makarow: Thernodynamische Lintersuchung des Systems NickelchloridManganchlorid-Wasser bei $25^{\circ} \mathrm{C}$. Mit 1 Abb. und 2 Tah. (Eing. 7. 2. 77; in rev. Fassg. 27. 10. 77)

A. B. ZAKI and S. E. MoRSI: Mass-transfer of $\mathrm{H}^{-} \mathrm{N}_{1}{ }^{2}$ - System in Thin layers of Macroreticular Ionexchange Resin. With 3 Fig. and 2 Tah. Received 2 5.77; in rev version 7. 12.77, 
A. Z. Trifonov and N. V. Pavlyukevich: A Spectroscopic Method for Investigating Transport Processes in Capillaries During Phase Conversion. With 4 Fig. and 2 Tab. (Received 11. 10. 77; in rev. version 20.12.77)

A. K. SRIvastava: Solid-State Reactions between Maleic Anhydride and Substituted Aromatic Amines. With 2 Fig. and 7 Tab. (Received 27. 5. 77; in rev. version 29. 12. 77) . ........6 6

V. F. Baibuz, V. Yu. Zitserman and L. M. Berezhrovskir: Optimization Method for Calculation of Associated Parameters. With 2 Fig. and 5 Tab. (Received 12. 12. 77) . . . . . . . . . .

M. S. A. Abd-El-Mottaleb and Z. H. Khalil: Electronic Spectra and Solvachromism of Some Cyanine Dyes. With 2 Fig. and 5 Tab. (Received 9. 9. 77; in rev. version 7. 1. 78) ........650

Z. LÁSzLó: Versuchsergebnisse zur Strofftrennung bei der Wechselstromelektroosmose. Mit 3 Abb. (Eing. 15. 10.77)

J. BENDIG und D. KREYSIG: Desaktivierungsverhaiten von Arenen und Heteroarenen. XIII. Das Extinktion-Zeit-Verhalten bei der Photodimerisierung. Mit 4 Abb. und 1 Tab. (Eing. 2. 2. 78) ...

1. LIEBERWIRTH und H. SCHUBERTH: Das isotherme Dampf-Flüssigkeits-Phasengleichgewichtsverhalten des Systems Aceton'Wasser bei $35^{\circ}$ C. Mit 1 Abb. und 1 Tab. (Eing. 11. 4. 78)

H.-D. IlGt: Bestimmung der UV VIS-Extinktionshoeffizienten in photochemisch aktiven Mehrkomponentensystemen (homogene Phase). Mit 4 .4bb. (Eing. 4. 10. 77; in rev. Fassg. 2. 2. 78) ..

W. Gründler, M. SchWanke. und H. Kohl.leppel: Modellrechnungen zur zwischenmolekularen Wechselwirkung von ungesättigten Kohlenwasserstoffen. Mit 5 Abb. (Eing. 25. 1. 78) ..... 686

F. Friedeamanv, V. GoliziN und W. Gründler: FSGO-Berechnungen an den Moleküldimeren $\mathrm{H}_{4}$, $\mathrm{Li}_{4}, \mathrm{Li}_{2} \mathrm{H}_{2}, \mathrm{Be}_{2} \mathrm{H}_{4}, \mathrm{Na}_{2} \mathrm{H}_{2}$ und $\mathrm{Li}_{2} \mathrm{~F}_{2}$. Mit $1 \mathrm{Abb}$. und $7 \mathrm{Tab}$. (Eing. 25. 1. 78) $\ldots \ldots \ldots \ldots 69$

R. V. Gopala Rao and A. K. Murthy: Three Body Correlations in Liquid Rubidium. With 2 Fig. (Received 2. 3. 78)

K. Geier und H.-J. Bittrich : Zur Thermodynamik der Flüssig-Dampf-Gleichgewichte der binãren Systeme n-Hexan/Benzol. Cyclohexan/Benzol und Tetrahydrofuran mit n-Hexan, Cyclohexan, Benzol und Dimethylformamid. Mit $11 \mathrm{Abb}$. und 4 Tab. (Eing. 25. 1. 78)

R. Wetzel, L. Müller und I. Babanskaja: Der Einfluß von Oberflächendotierungen auf die elektrokatalytische Aktivität von Ag-Elektroden bei der anodischen Oxydation von $\mathrm{HCHO}$ in alka-

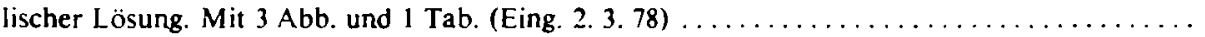

R. SChöll.Ner, W. JaCOB und H.-G. MALZ: Untersuchungen zur Bestimmung von Kationenpositionen in Zeolithen vom Faujasittyp. I. $\mathrm{K}^{+}$-Positionen in $\mathrm{NaKMe}^{2+}$ A-Zeolithen. Mit $11 \mathrm{Abb}$. (Eing. 8. 3. 78)

G. Heivrich. E. Straube und G. Helmis: Zur Theorie der Konfigurationsbehinderungen in polymeren Netzwerken. Teil I: Das „Gedächtnis“-Oszillator-Modell. (Eing. 25. 1. 78)

R. ZIEBIG und F. Pragst: Elektrochemische Erzeugung von Triplettzuständen. VII. Triplettenergieübertragung in der elektrochemischen Lumineszenz. Mit 6 Abb. und 3 Tab. (Eing. 8. 2. 78)

V. A. Tatarinov, K. Gloe, I. M. Ivanoi, P. I. Vasad. P. Mühl und L. M. Gindin: Zum Temperatureinfluß auf die Extraktion von Cobalt(II)-chlorid mit Tetraoctylammoniumchlorid. Mit 2 Abb. und 2 Tab. (Eing. 15. 3. 78)

R. PATZ und M. T. RätZSCH: Kerrkonstanten flüssiger Systeme. IV. Untersuchungen zum Kontinuumsmodell von Scholte. Die Berechnung der Temperatur- und Konzentrationsabhängigkeit der Kerrkonstanten. Mit 13 Abb. und 4 Tab. (Eing. 24. 12. 77; in rev. Fassg. 19. 3. 78)

R. Patz und M. T. Rätzsch: Kerrkonstanten flüssiger Systeme. V. Untersuchungen zum Kontinuumsmodell von Scholte. Modifizierte molare Kerrkonstanten birärer Mischung. Mit 2 Tab.

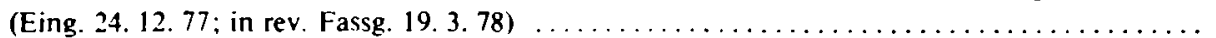


R. Zrearg und F. Praast: Elektrochemische Erzeugung von Triplettzuständen. VIII. Zur elektrochemischen Lumineszenz von Isobenzofuranen. Mit 3 Abb. und 4 Tab. (Eing. 31. 3. 78) ....

\section{Kurze Mitreilungen}

E. MÜller, H. Matschiner und H.-D. DörfleR: Elektrosorptions- und Inhibitionseigenschaften von Triphenyl-phosphonium-āthylbromid. Mit 4 Abb. und 1 Tab. (Eing. 13. 7. 78) ..... 804

J. Westfahl, P. Jantetz und R. Dehmlow: EinfluB experimenteller Bedingungen auf das Flach-

\section{Heft 5}

Ausgegeben im Oktober 1979

H. SCHÄFER und W. Schmor : Ligandeneffekte auf die Reaktionen von Triplett-Methylenblau und Triplett-Thionin mit Fe II. Mit 4 Abb. und 3 Tab. (Eing. 2. 12.77; in rev. Fassg. 24. 4. 78) . . . 817

S. C. Pati and Y. SRiramulu: Kinetics and Mechanism of the Ruthenium(lil) Chloride Cataly sed Oxidation of Phenol and Substituted Phenols by Periodate in Aqueous Alkaline Medium. $W$ ith 3 Fig. and 4 Tab. (Received 10. 3. 78)

R. NikouIć and H. A. ØYE: Co(II) Spectra in Molten Mixtures of r-Picolınium (hloride-Aluminium Chloride. With 4 Fig. and 2 Tab. (Received 25. 1. 78)

K. Bhatt and C. K. Nand: Kinetics and Mechanism of Reduction of Cerium(IV) by Ascorbic Acid. With 7 Fig. and 7 Tab. (Received 20.1.78)

H. Schäfer, R. Stahn und W. Schmidt: Lösungsnitteleffekte auf die Fluoreszenz-Quantenausbeuten von Thionin und Methylenblau. Mit $10 \mathrm{Abb}$. und $3 \mathrm{Tab}$. (Eing. 29. 4. 78)

M. Gutmann, I. Schober und D. Haberland: Thermokinetische Untersuchung der Reaktion von Pentacyanokobaltat(II) mit organischen Halogenverbindungen. II. Auswertung der experimentellen Ergebnisse mit Hilfe eines Iterationsverfahrens zur Restimmung de; Gesamtintegrah $\infty$

$\int_{0}^{\infty} \Delta T \mathrm{~d} t$. Mit $5 \mathrm{Abb}$. und $1 \mathrm{Tab}$. (Eing. 30. 1. 78)

W. LORENZ, C. ENGLer und M. Kretschmer: L'ntersuchung von MO-Parametern zur Berechnung lokaler Potentialfächen und Ladungen in großen Systemen. Mit $1 \mathrm{Abb}$. und $3 \mathrm{Tab}$. (Enng 11. 4. 78)

H. Schäfer und W. Schmidt: Lösungsmitteleffekte auf die Photorcaktionen ion Methylentlat und Thionin mit Fe II. Mit 7 Abb. und 4 Tab. (Eing. 29. 4. 78)

R. Harlfinger, D. Hoppach, H.-P. Hofmanin und K. QuirzsCh Zum Adsorptionsverhalten ion $\mathrm{C}_{\mathbf{4}}$-Kohlenwasserstoffen an synthetischen Zeolithen der Faujasitstruktur. 1. Gravimetrische Untersuchungen des Adsorptionsgleichgewichtes. Mit 6 Abb. und 3 Tab. (Eing, 18. 4. 78) , .. 905

H. DUNkeN und H. Hobert: Halbempirische quantenchemische Berechnungen an Oxıd- und Silikatoberflächenklustern. Mit $6 \mathrm{Abb}$. und 8 Tab. (Eing. 11.4. 78)

S. A. KrRILlov und W. Horlbeck: Die Umorientierung der Nitrationen in festen und flussigen Nitraten einwertiger Metalle. Mit 1 Abb. und 3 Tab. (Eing. 15.4. 78) ........... . . 931

U. EberSbach, C. Schürer und C. Weissmantel: Zur Korrosionsbeständigkeit von tonenplatticrten Kohlenstoffschichten auf Eisen- und Stahlsubstraten. M16 5 Abb. (Eing. 4. 12.78; ir. ra. Fassg. 21. 12. 78) 
K. Fiedler und H.-J. SPANGenberg: Monte-Carlo-Berechnung thermodynamischer Funktionen langkettiger Normalparaftine. Mit 1 Abb. und 3 Tab. (Eing. 10. 4. 78)

M. Gutmann, I. Schober und D. HaberLand: Thermokinetische Untersuchung der Reaktion von Pentacyanokobaltat(II) mit organischen Halogenverbindungen. III. Kinetische Untersuchung der Temperatur- und Lösungsmittelabhängigkeit von Reaktionen des $\left[\mathrm{Co}(\mathrm{CN})_{5}\right]^{3-}$ mit organischen Halogenverbindungen. Mit $4 \mathrm{Abb}$. und 5 Tab. (Eing. 3. 4. 78) .

\section{Kurze Mitteilungen}

S. C. Ameta, P. N. Pande and K. L. Dayma: Correlation Analysis in Paper Chromatography-III2,4-dinitrophenylhydrazides of Fatty Acids. With 1 Fig. and 1 Tab. (Recsived 16. 11.78) .....

A. V. Sarma, S. N. Rao and K. S. Rao: Ultrasonic Study in Critical Systems of O-Chloraniline with Iso-Octane and n-Heptane. With 2 Fig. and 2 Tab. (Received 16. 3. 78)

K. Altenburg: Zur Berechnung der quadratischen Mittelwerte der Radien größerer isomerer

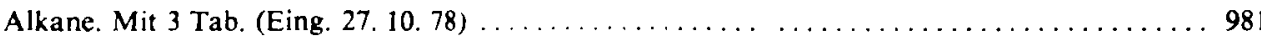

H. Kehlen, F.-U. Schneider und P. Hörcher: Zur Berechnung der freien Exzeßenthalpie aus binären eutektischen Schmelzgieichgewichten. Mit 1 Tab. (Eing. 8. 12. 78) $\ldots \ldots \ldots \ldots \ldots \ldots \ldots 98$

R. Radecilia: Die Gewinnung von Strukturinformation aus Aufspaltungen zweiter Ordnung in ${ }^{1} \mathrm{H}$-CW-off-resonance entkoppelten ${ }^{13}$ ('-NMR-Spektren. Mit 3 Abb. (Eing. 14. 12. 78) ..... 990

L. Müller: Úber den Zusammenhang zw'ischen den in Gleichgewichtsnähe und aus der Tafelneigung bestimmten Durchtrittskoeffizienten. (Eing. 5. 2. 79)

L. MÜLleR und B. KaISER: Untersuchungen zur Kinetik der Chlorentwicklung und -reduktion an iridiumbedeckten Titanelektroden. Mit $4 \mathrm{Abb}$, und $1 \mathrm{Tab}$. (Eing. 5. 2. 79) . ............ 999

H.-J. Bittrich, G. ZimmermanN und E. SChaAR: Untersuchungen zur selektiven Stofftrennung. VI. Orthoester als Selektivlösungsmittel für Kohlenwasserstoffırennungen. Mit $3 \mathrm{Tab}$. (Eing.

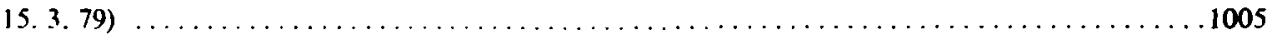

H.-J. Bittrich, H. Gedan und G. Feix: Zur Lóslichkeitsbeeinflussung von Kohlenwasserstoffen in

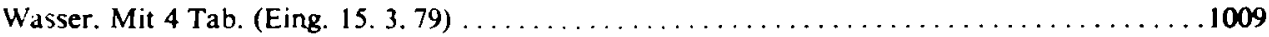

H.-J. BitTrich: Bemerkung en zu der Mitteilung von L. MülLER ,Thermodynamische Begründung der Gültigkeit der linearen freien Enthalpiebeziehungen für beliebige chemische Reaktionen *.

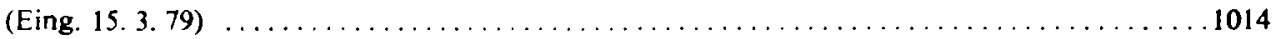

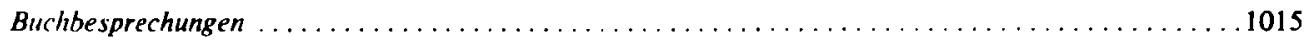

\section{Heft 6}

Ausgegeben im Dezember 1979

A. B. ZAKı: Kinetics of the Ion-Exchange $\mathrm{S}_{\mathrm{j}} \mathrm{stem}\left[\mathrm{Fe}\left(\mathrm{SO}_{4}\right)_{2}\right]^{-} / \mathrm{SO}_{4}^{2-}$ in a Strong-Base Anion Ex-

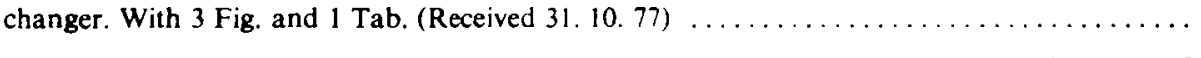

V. Shanmugasundaram and P. Thiyagarajan: Anisotropy of the Molecular Polarizability of Hydrocarbons Cycloalkenes, Alcohols and Alkyl Halides. With 1 Tab. (Received 17. 11. 77) . 1023

M. Borówko, M. Jaroniec and W. RudzińSk1: Adsorption from Multicomponent Mixtures on Heterogeneous Solid Surfaces. With 1 Fig. (Received 23. 1. 78) $\ldots \ldots \ldots \ldots \ldots \ldots \ldots \ldots 1027$

F. Mata and M. S. Crespo: Catalytic Polarographic Prewaves. I. The Ni(II)-pyridine System. With 5 Fig. and 3 Tab. (Received 15. 12. 77; in rev. version 5. 4. 78) $\ldots \ldots \ldots \ldots \ldots \ldots \ldots 1033$ 
F. Mata and M. S. Crespo: Catalytic Polarographic Prewaves. II. The Ni(lI)-o-phenylene Diamine System. With 6 Fig. and 3 Tab. (Received 5. 4. 78)

G. Henrion, D. Marquardt und B. Stöcker: Bestimmung der Halogene durch Flammenemis-

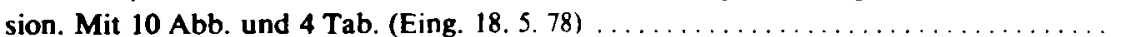

T. OJkova: Uber die Veränderung der freien Mischungsenthalpie $\left(1 G^{f}\right)$ bei der Bildung von festen Lösungen-Siebenhydraten. Mit $3 \mathrm{Abb}$. und 3 Tab. (Eing. 17. 4. 78)

J. Vavra and A. Rosenbergerova: Light Scattering in Mixtures of Pyridine with Formic. Ace:ic and Propionic Acids. With 4 Fig. (Received 4. 5. 78)

M. BoHL: CNDO-Berechnungen der Potentialkurven für die chemisorptıve Bindung von $\mathrm{O}$ an $\mathrm{Yi}$ Clustern mit (100)-Oberfläche. Mit 2 Abb. und 2 Tab. (Eing. 4. 2. 78; in rev. Fassg. 6. 5. -8)

A. Zikánová, M. Kočiřik, M. Bülow, J. Kärger und A. Varhaniková: lintersuchung der Sorptionskinetik von Cyclohexan an bindemittelfreien NaX-Zeolith-Preßlıngen. Mit $6 \mathrm{Ab}$ b. und 1 Tab. (Eing. 13.6. 78)

W. Rudziński, J. Narkiewicz and A. Patrykiejew: Effects of Local Correlations between tdsorbed Molecules in the Localized Adsorption of Gases on Patchwise Heterogeneous Surfaces. With 2 Fig. and 1 Tab. (Received 11. 5. 78)

H.-D. ILgE: Zur grafischen Extinktionsau!swẹtung UV VIS-spektroskopisch verfolgter Reaktionen in Systemen mit mindestens vier Komponenten heim Ablauf von drei linear unabh.̈ngigen Teilreaktionen. Mit 11 Abb. und 7 Tab. (Eing. 3. 5. 78) .

H. KResse, H. SackmanN und H. Kehlen: Das Verhalten binärer füssiger Mischphasen. 11. Mitt. Das dielektrische Verhalten von Systemen aus Cyclohesan und Tetrachloriden. Mit 5 Tab. (Eing. 25. 1. 78; in rev. Fassg. 11. 5. 78)

E. Вich, G. Opel, R. Pietsch und E. Vociel: Der zweite V'irialkoeffizient und der Viskositjtskoeffizient von Benzoldampf und ihre I)arstellbarkeit durch Lennard-Jones-(m-n)-Ansätze für das intermolekulare Potential. Mit 9 Tah. (Fing. 8. 6. 78)

G. KolláR: Korrelationsverfahren zur Berechnung von Dampfdruckwerten auf (irund der molproportionalen ebullioskopischen Konstanten. Mit 4 Tab. (Eing. 24. 1. 78)

B. Michel: A Note on Spinodal Decomposition near Interfaces. With 3 Fig. (Received 11. 5. -8) 1165

H.-D. DörfLER und E. MülLER: Elektrokapillaritätsuntersuchungen an Dodekanolpolyglyholäthern in methanolischen Elektrolytlösungen. Mit 5 Abb. und 1 Tab. (Eing. 27. 5. 78) .

H. Herden, R. Broddack und R. Schöllnt R: Zum Transportverhalten der n-Butene an Zeolıthen des Typs NaZnA. Mit 5 Abb. und 7 Tab. (Eing. 17. 2. 78: in rev. Fassg. 31. 5. 78)

N. K. Sankhla, C. P. Gupta and R. K. Mehta: Physico-chemical Investigation of the Veral Chelates of o-(N-2-furfuralideneimino)-ethane Sulphonic Acid with $\mathrm{Cr}(\mathrm{II})$, Mn(II). Fetill, $\mathrm{Co}(\mathrm{II}), \mathrm{Ni}(\mathrm{II})$ and $\mathrm{Cu}(\mathrm{II})$. With $1 \mathrm{Fig}$. and 2 Tab. (Received 29.5 781 .

A. Szymański, A. Podgórski and A. Huczko: Decomposition and Reduction of Silicon DiNMc in a Low-Temperature Plasma Jet. With 3 Fig. and 3 Tab. (Recersed 6. 6. 78)

\section{Kurze Mitteilung}

W. Hopf: Der dynamische Randwinkel unter besonderer Beachtung der Adsorption von flh!laminen an festen Quarz- oder Glasgrenzflächen (Eing. 20 3. 7.

Buchbesprechungen

Anhang

Sachregister und Autorenregister zu Band 259 (1978) 

